\title{
ON THE SOLVABILITY OF SEMILINEAR OPERATOR EQUATIONS AND ELLIPTIC BOUNDARY VALUE PROBLEMS
}

\author{
BY MELVYN S. BERGER ${ }^{1}$ AND MARTIN SCHECHTER ${ }^{1}$ \\ Communicated by Hans F. Weinberger, February 25, 1972
}

Let $L$ be a bounded linear Fredholm mapping of index zero, mapping a Banach space $X$ into a Banach space $Y$. Then necessary and sufficient conditions for the solvability of the operator equation $L u=f$ for $f \in Y$ are well known. However the same satisfactory state of affairs does not hold for semilinear operator equations in which a compact nonlinear operator $N u$ is added to the right hand side of $L u=f$. Indeed, most recent approaches to this problem attempt to place enough restrictions on the operator $N$ so that $L-N$ is surjective.

A similar state of affairs holds in the case of elliptic boundary value problems defined on bounded domains $\Omega \subset R^{N}$. Recently, however, in the special case of the semilinear Dirichlet problem,

$$
\Delta u+\lambda_{1} u+h(x, u)=f,\left.\quad u\right|_{\partial \Omega}=g(x), \quad|h(x, u)| \leqq M,
$$

where $\lambda_{1}$ denotes the smallest eigenvalue of the Laplacian $\Delta$ on $\Omega$ Landesmann and Lazar [1] found interesting necessary and sufficient conditions for solvability that reduce to the usual orthogonality restrictions when $h(x, u) \equiv 0$. In [2], Nirenberg generalized the sufficiency conditions of [1] to semilinear operator equations of the form $L u=N u+f$, where $L$ is a Fredholm map of index $p \geqq 0$ of a Banach space $X$ into itself, and $N$ is a compact map of $X$ into itself. He also applied his result to the solvability of a large class of semilinear elliptic boundary value problems. However the conditions obtained by Nirenberg are not necessary in general, and in addition require the computation of topological invariants for their applicability. See also [3].

It is the purpose of this paper to provide computable necessary and sufficient conditions for the solvability of a class of semilinear problems intermediate between those of [1] and [2]. The conditions we obtain, on the one hand, can be considered as extensions of orthogonality to a nonlinear context; and on the other hand, are independent of topological considerations.

1. The Hilbert space problem. Let $H$ be a Hilbert space over the reals,

AMS 1970 subject classifications. Primary 47H15, 35J60.

${ }^{1}$ Research partially supported by an NSF grant.

Copyright (C) American Mathematical Society 1972 
and suppose $L$ is a bounded selfadjoint linear operator of $H$ into itself that is Fredholm with no negative essential spectrum. We also suppose that $N u$ is a $C^{1}$ gradient operator defined on $H$, so that there is a $C^{2}$ realvalued function $\mathscr{N}(u)$ defined on $H$ such that $\mathscr{N}^{\prime}(u)=N u$, i.e. the Fréchet derivative of $\mathcal{N} u$ is $N u$ for all $u \in H$. We wish to determine necessary and sufficient conditions for the solvability in $H$ of the operator equation

$$
L u=\mathscr{N}^{\prime}(u)+f .
$$

An immediate necessary condition is that

$$
\left(\mathcal{N}^{\prime}(u), w\right)+(f, w)=0 \quad \text { for all } w \in \operatorname{Ker} L .
$$

We shall determine conditions on $\mathscr{N}(u)$ such that (3) is also sufficient for the solvability of (2). A result in this direction is

THEOREM 1. Suppose the $C^{2}$ functional $\mathcal{N}(u)$ satisfies the following properties: (i) convexity on $\mathrm{H}$; (ii) continuity with respect to weak convergence in $H$; (iii) as $\|u\| \rightarrow \infty \mathscr{N}(u) \leqq(c / 2-\varepsilon)\|u\|^{2}$, where $\varepsilon>0$ and $c$ is the smallest positive point in the spectrum of $L$; (iv) $\left(\mathcal{N}^{\prime}(w+z), z\right)-\mathscr{N}(w+z)$ $\rightarrow \infty$ as $\|z\| \rightarrow \infty(z \in \operatorname{Ker} L)$ uniformly over bounded sets $\|w\| \leqq M$ in $H$; (v) $\mathcal{N}^{\prime \prime}(u)$ injective on Ker $L$ for every $u$. Then a necessary and sufficient condition for the solvability of (2) is the existence of a $u \in H$ such that (3) holds.

Proof. For brevity, suppose $(L u, u) \geqq 0$ for all $u \in H$.

First we show that under the above hypotheses on $\mathcal{N}(u)$, the critical points of the functional

$$
I(u)=\frac{1}{2}(L u, u)-\mathscr{N}(u)-(f, u)
$$

under the constraint (3) are in (1-1) correspondence with the solutions of (2). Let $\left(w_{1}, \ldots, w_{N}\right)$ be an orthonormal basis for $\operatorname{Ker} L$, so that (3) is equivalent to

$$
\left(\mathcal{N}^{\prime}(u), w_{i}\right)+\left(f, w_{i}\right)=0 \quad(i=1, \ldots, N) .
$$

Thus the critical points just mentioned satisfy the equation

$$
L u=\mathcal{N}^{\prime}(u)+f+\sum_{i=1}^{N} k_{i} \mathcal{N}^{\prime \prime}(u) w_{i},
$$

where the $k_{i}$ are real numbers. We shall show that $\sum_{i=1}^{N} k_{i} \mathcal{N}^{\prime \prime}(u) w_{i}$ $=\mathscr{N}^{\prime \prime}(u) \bar{w}=0$ where $\bar{w}=\sum_{i=1}^{N} k_{i} w_{i}$. Taking inner products of each $w_{j}$, with $(6)(j=1, \ldots, N)$, we find, by virtue of $(3)$, that $k_{1}, \ldots, k_{N}$ satisfy the linear equations 


$$
\sum_{i=1}^{N} k_{i}\left(\mathcal{N}^{\prime \prime}(u) w_{i}, w_{j}\right)=0 \quad(j=1, \ldots, N) .
$$

Since $\mathscr{N}$ is a $C^{2}$ convex function, $\mathscr{N}^{\prime \prime}(u)$ is a selfadjoint nonnegative linear operator. Now (7) implies that $\left(\mathcal{N}^{\prime \prime}(u) \bar{w}, \bar{w}\right)=0$; hence $\mathcal{N}^{\prime \prime}(u) \bar{w}=0$. As $\mathcal{N}^{\prime \prime}(u)$ is injective, $\bar{w}=0$.

Next we prove the existence of an absolute minimum $I_{0}>-\infty$ for (4). Now any element $u \in H$ can be uniquely written as $u=v+z$ where $z \in \operatorname{Ker} L$ and $v \in[\operatorname{Ker} L]^{\perp}$, so that the selfadjointness and positivity of $L$ imply the existence of an absolute constant $c>0$ such that $(L v, v) \geqq c\|v\|^{2}$. In addition, the convexity of $\mathscr{N}(u)$ implies that $\mathscr{N}(v) \geqq \mathcal{N}(v+z)$ $-\left(\mathcal{N}^{\prime}(v+z), z\right)$. Consequently, if $u$ satisfies (3), we find, from (4),

$$
\begin{aligned}
I(v+z) & =\frac{1}{2}(L v, v)-\mathscr{N}(v+z)-(f, v)-(f, z) \\
& \geqq \frac{c}{2}\|v\|^{2}-\mathscr{N}(v+z)-(f, v)+\left(\mathcal{N}^{\prime}(v+z), z\right) \\
& \geqq \frac{c}{2}\|v\|^{2}-(f, v)-\mathscr{N}(v) .
\end{aligned}
$$

Now hypothesis (iii) implies that $I(v+z) \rightarrow \infty$ as $\|v\| \rightarrow \infty$, as well as that $\inf _{H} I(u) \geqq I_{0}>-\infty$.

Finally we show that inf $I(u)$ over the class defined by (3) is attained by an element $u_{0}$ satisfying (3). Since the functional $I$ is differentiable, $u_{0}$ will satisfy (2) and (3). Hypothesis (ii) on $\mathcal{N}(u)$, and (8), imply that $I(u)$ is lower semicontinuous with respect to weak convergence in $H$. Therefore it suffices to prove that (a) the set $S=\left\{u \mid I(u) \leqq I_{0}+1\right\} \cap \Sigma$ is uniformly bounded in $H$ where $\Sigma$ is the set satisfying (3), and (b) $\Sigma$ is weakly closed. Since $I(v+z) \rightarrow \infty$ as $\|v\| \rightarrow \infty$ by (10), to prove (a) it suffices to show that $I(v+z) \rightarrow \infty$ as $\|z\| \rightarrow \infty$ and $\|v\| \leqq M$. However, by virtue of hypothesis (iv), this fact follows immediately from (9).

Lastly, we demonstrate (b): The set $\Sigma$, of elements $u \in H$ satisfying (3), is weakly closed. We note that the smoothness of $\mathcal{N}(u)$ and hypothesis (ii) imply that $\mathscr{N}^{\prime}(u)$ maps weakly convergent sequences into strongly convergent sequences. Consequently, if a sequence $\left\{u_{n}\right\}$ satisfies (3) and converges weakly to $u \in H, u$ also satisfies (3). Thus $\Sigma$ is weakly closed; and the theorem is proved.

RemarKs. 1. Taylor's theorem applied to the functional $\mathcal{N}(u)$ shows that hypothesis (iv) is satisfied if, for example, $\left(\mathcal{N}^{\prime \prime}(v+z) z, z\right) \rightarrow \infty$ as $\|z\| \rightarrow \infty(z \in \operatorname{Ker} L)$ uniformly for $\|v\| \leqq$ const.

2. Necessary and sufficient conditions for the solvability of (2) analogous to those of Theorem 1 can be proven if (iii) is weakened to $\lim \mathcal{N}(u) /\|u\|^{2}$ 
$<\infty$ as $\|\mathrm{u}\| \rightarrow \infty$. Moreover for $L$ nonnegative (with the notation defined above) such growth restrictions can be removed by replacing hypotheses (iii) and (iv) above with: (iii)' whenever $u=v+z$ satisfies (3) and $\|v\| \rightarrow \infty$ there are absolute constants $c_{1}, \varepsilon>0$ such that

$$
\left(\mathscr{N}^{\prime}(v+z), z\right)-\mathscr{N}(v+z) \geqq-c_{1}-\left(\frac{1}{2} c-\varepsilon\right)\|v\|^{2} .
$$

2. An application to semilinear elliptic boundary value problems. Let $x$ denote a point in a bounded domain $\Omega \subset \boldsymbol{R}^{N}$. Suppose

$$
L u=\sum_{0 \leqq|\alpha|,|\beta| \leqq m}(-1)^{|\alpha|} D^{\alpha}\left\{a_{\alpha \beta}(x) D^{\beta} u\right\}
$$

is a formally selfadjoint elliptic differential operator of order $2 m$ with smooth coefficients. It follows that the solutions of the linear Dirichlet problem

$$
L u=0 \quad \text { in } \Omega,\left.\quad D^{\alpha} u\right|_{\partial \Omega}=0, \quad|\alpha| \leqq m-1,
$$

form a finite-dimensional vector space; while the quadratic form

$$
\sum_{|\alpha|+|\beta|=2 m} \int_{\Omega} a_{\alpha \beta}(x) D^{\beta} u D^{\alpha} u>0 \text { for } u \in C_{0}^{\infty}(\Omega)(u \neq \equiv) .
$$

Then as an application of Theorem 1, we determine necessary and sufficient conditions for the solvability of the semilinear elliptic boundary value problem

$$
L u=g(x, u)+f \quad \text { in } \Omega,\left.\quad D^{\alpha} u\right|_{\partial \Omega}=0, \quad|\alpha| \leqq m-1 .
$$

THEOREM 2. Suppose the function $g(x, u)$ is continuously differentiable and satisfies the following conditions:

(i) the partial derivative $g_{u}(x, u) \geqq 0(b u t \not \equiv)$;

(ii) for $0<b<\lambda_{1}^{*}$ (the first positive eigenvalue of $L$ ), $|g(x, u)| \leqq b|u|$ as $|u| \rightarrow \infty$;

(iii) $\int_{0}^{s} u g_{u}(x, u) d u \rightarrow \infty$ as $|s| \rightarrow \infty$ uniformly in $x$.

Then a necessary and sufficient condition for the solvability of (12) is the existence of a function $u \in \dot{W}_{m, 2}(\Omega)$ such that $\int_{\Omega}[g(x, u) w+f w]=0$ for all $w$ satisfying (11).

A useful example can be obtained by choosing $g(x, u)=\arctan u$. We also note that the boundedness restriction (ii) of Theorem 2 can be considerably weakened by virtue of the Remark 2 above, and the extended theorem yields some sharp recent results on the existence of conformal metrics of prescribed Gauss curvature.

\section{BIBLIOGRAPHY}

1. E. M. Landesmann and A. C. Lazer, Nonlinear perturbations of linear elliptic boundary value problems at resonance, J. Math. Mech. 19 (1970), 609-623. MR 42 \#2171. 
2. L. Nirenberg, An application of generalized degree to a class of nonlinear problems, Troisième Colloq. Anol. Fonct. Liège Centre Belge de Recherches Mathématiques, 1971, pp. 57-73.

3. S. A. Williams, A sharp sufficient condition for solution of a nonlinear problem, J. Differential Equations (1970), 580-586.

Department of Mathematics, Belfer Graduate School, Yeshiva University, NEW YORK, NEW YORK 10033

Current address: (M. S. BERGER) DEPARTMENT OF MATHEMATICS, INSTITUTE FOR AdVANCED Study, PrinCETON, NeW JERSEY 08540 